\title{
An immunohistochemical study of the distribution of carcinoembryonic antigen in epithelial tumours of the ovary
}

\author{
J. HEALD, C. H. BUCKLEY, AND H. FOX \\ From the Department of Pathology, University of Manchester, UK
}

SUMmaRY An immunohistochemical study of the tissue CEA content of 82 epithelial neoplasms of the ovary has shown that mucinous tumours contain more of this substance than do their serous counterparts; otherwise a knowledge of tissue CEA content appears to be of little value in the differential histological diagnosis of this group of neoplasms.

Among mucinous tumours there is only a partial correspondence between their degree of malignancy, as assessed histologically, and their content of CEA. It is postulated that immunohistological study of tissue CEA may add a degree of finesse to morphological analysis of these neoplasms and thus allow for a more precise grading of their degree of malignancy.

Carcinoembryonic antigen (CEA), first identified in the plasma of patients with colonic adenocarcinoma (Gold and Freedman, 1965a) and initially believed to be a specific product of fetal gut and of tumours of endodermal origin (Gold and Freedman, 1965b), is now known to be present in the plasma of individuals with a wide range of benign and malignant conditions (Lo Gerfo et al., 1971; Reynoso et al., 1972; Pusztaszeri and Mach, 1973; Hansen et al., 1974). There have been a number of reports of elevated plasma CEA levels in women with ovarian neoplasms (Khoo and Mackay, 1973; van Nagell et al., 1975a, 1975b; DiSaia et al., 1975; Barrelet and Mach, 1975; Samaan et al., 1976; Puri et al., 1977) but the more recently introduced immunoperoxidase method for the demonstration of CEA in tissue sections (Primus et al., 1975; Goldenberg et al., 1976; Isaacson and Le Vann, 1976; Heyderman and Neville, 1977) has not been extensively applied to ovarian tumours. Marchand et al. (1975), using this technique, were unable to detect any CEA in serous tumours and demonstrated this antigen only in those mucinous neoplasms in which the epithelium was of the 'intestinal' type while van Nagell et al. (1978), who studied 88 ovarian carcinomas, found CEA in $21 \%$ of those of mucinous type and in only $2 \%$ of the serous tumours. Rutanen et al. (1978) could, by contrast, detect no CEA in any of the 12 ovarian

Received for publication 31 January 1979 carcinomas that they studied though the antigen was present in two of seven mucinous cystadenomas.

The present study was undertaken partly because of the discrepancies in these previous reports and partly to attempt to answer two specific questions: (i) Is the presence of CEA in an ovarian epithelial neoplasm dependent upon, or related to, the specific nature of the tumour epithelium, its presence or absence being thus of value in the differential histological diagnosis of this group of neoplasms? (ii) In epithelial tumours of the ovary, in which there is a continuous spectrum from fully benign to frankly malignant, is there any correlation between the degree of malignancy of any particular neoplasm and its tissue content of CEA?

\section{Material and methods}

Formalin-fixed, paraffin-processed tissue from specimens received in the pathological laboratory at St Mary's Hospital, Manchester were used in this study. The series comprised 82 epithelial neoplasms of the ovary (Table), and in each case eosin and haematoxylin stained sections were examined and a consensus histological diagnosis was agreed, using for this purpose the criteria laid down by the World Health Organisation (Serov and Scully, 1973). Mucinous tumours were also stained by Bodian's protargol method and those which contained argyrophil cells were classed as being of 'intestinal' type (Fox et al., 1964). 
Table Ovarian neoplasms studied for CEA content

\begin{tabular}{lllr}
\hline Type of tumour & $\begin{array}{l}\text { Total } \\
\text { no. }\end{array}$ & Histological grading & No. \\
\hline Mucinous & 30 & Benign & 10 \\
& & Borderline & 11 \\
Serous & 36 & Malignant & 9 \\
& & Benign & 9 \\
Endometrioid & 12 & Malignant & 7 \\
Mesonephroid & 4 & Malignant & 20 \\
& & Malignant & 12 \\
\hline
\end{tabular}

Serial $5 \mu \mathrm{m}$ sections were cut from each block of tissue and treated with anti-CEA serum to determine the highest dilution at which staining could be detected. All the antisera used were commercially prepared (Dakopatts), and for the demonstration of CEA a modification of the unlabelled antibody enzyme (PAP) method (Sternberger et al., 1970) was used. Sections were dewaxed in xylene and then submerged in a $1 \%$ solution of 100 volume hydrogen peroxide in methanol for 45 minutes to abolish endogenous peroxidase activity. The non-specific background staining was reduced by treating the sections with non-immune non-conjugated swine serum in buffer at a dilution of 1 in 5 for 5 minutes; it was further reduced by bringing all the antisera to the required dilution in a 1 in 20 solution of swine serum in buffered saline. The non-immune swine serum was tipped off the slides and the sections were wiped, but not washed, before the appropriate dilution of rabbit anti-CEA antiserum was applied for 45 minutes. The sections were then treated with a 1 in 20 dilution of swine anti-rabbit-IgG antiserum for 15 minutes followed by a 1 in 60 dilution of rabbit peroxidase-antiperoxidase antiserum for 15 minutes. After each of the three stages the sections were rinsed briefly with two changes of tris buffered saline and then washed for 5 minutes over a magnetic stirrer. The staining was developed for 5 minutes in a freshly prepared solution of $0.035 \% \mathrm{w} / \mathrm{v}$ diaminobenzidine tetrahydrochloride (Sigma) and $0.01 \% \mathrm{w} / \mathrm{v}$ hydrogen peroxide in tris buffered saline (Graham and Karnovsky, 1966). Finally, the sections were washed in running water for 5 minutes, weakly counterstained with Harris's haematoxylin, dehydrated, and mounted.

Control sections of tumour tissue were treated with anti-CEA antiserum conjugated with purified CEA (British Standards Institute), and this resulted in complete abolition of staining (Figs 1a and 1b), as did also the substitution of non-immune rabbit serum for the specific anti-CEA antiserum. As a positive control, sections of a colonic adenocarcinoma known to contain CEA were stained.
Results

Staining of the cell surface or the glycocalyx was observed in a number of tumours but it was found that more consistent and easily assessed results were obtained if only the cytoplasmic reaction was evaluated. This also eliminated the difficulty of deciding whether stained material in a glandular lumen lay truly within the lumen or in the glycocalyx.

In tumours showing a positive reaction for CEA there was commonly a diffuse, uniform cytoplasmic staining of the cells containing this substance (Figs 2 and 3); in some cells, however, staining was concentrated in either the apical (Fig. 4) or basal (Fig. 5) regions while in a proportion of the mucinous neoplasms the staining was limited to the perinuclear cytoplasm (Fig. 6).

\section{MUCINOUS TUMOURS (Fig. 7)}

These were stained with the primary antiserum in dilutions ranging from 1 in 100 to 1 in 4000 . A positive reaction was obtained in only three $(30 \%)$ of the benign mucinous cysts, even at the lowest dilution of antiserum (1 in 100); within this group no staining was seen at a dilution of 1 in 800 . By comparison, all mucinous tumours of borderline malignancy showed some degree of positive staining at dilutions of 1 in 100 , though at dilutions greater than this the proportion of borderline tumours giving a positive reaction decreased progressively; nevertheless, at a 1 in 4000 dilution $45 \%$ still stained positively. In this group of tumours of borderline malignancy CEA was present in both the obviously proliferating areas and in those areas showing no atypical features.

All the mucinous carcinomas stained positively at a dilution of 1 in 3000 , and at a dilution of 1 in 4000 a positive reaction was still obtained in $78 \%$; no correlation was apparent between the tissue content of CEA and the degree of differentiation in this malignant group of neoplasms.

Mucinous tumours of 'intestinal' type were represented in the benign, borderline, and malignant groups, and these could not be separated out on the basis of their content of CEA, such neoplasms showing a staining pattern identical with those of non-intestinal type.

\section{SEROUS TUMOURS (Fig. 8)}

None of the benign serous tumours gave a positive reaction for CEA, even at a dilution of only 1 in 100 ; by contrast, $86 \%$ of the serous tumours of borderline malignancy and $80 \%$ of serous adenocarcinomas were positive for CEA at this dilution. These proportions fell with increasing dilution of the antiserum until at a dilution of 1 in 600 all the borderline tumours and $80 \%$ of the carcinomas failed to stain 


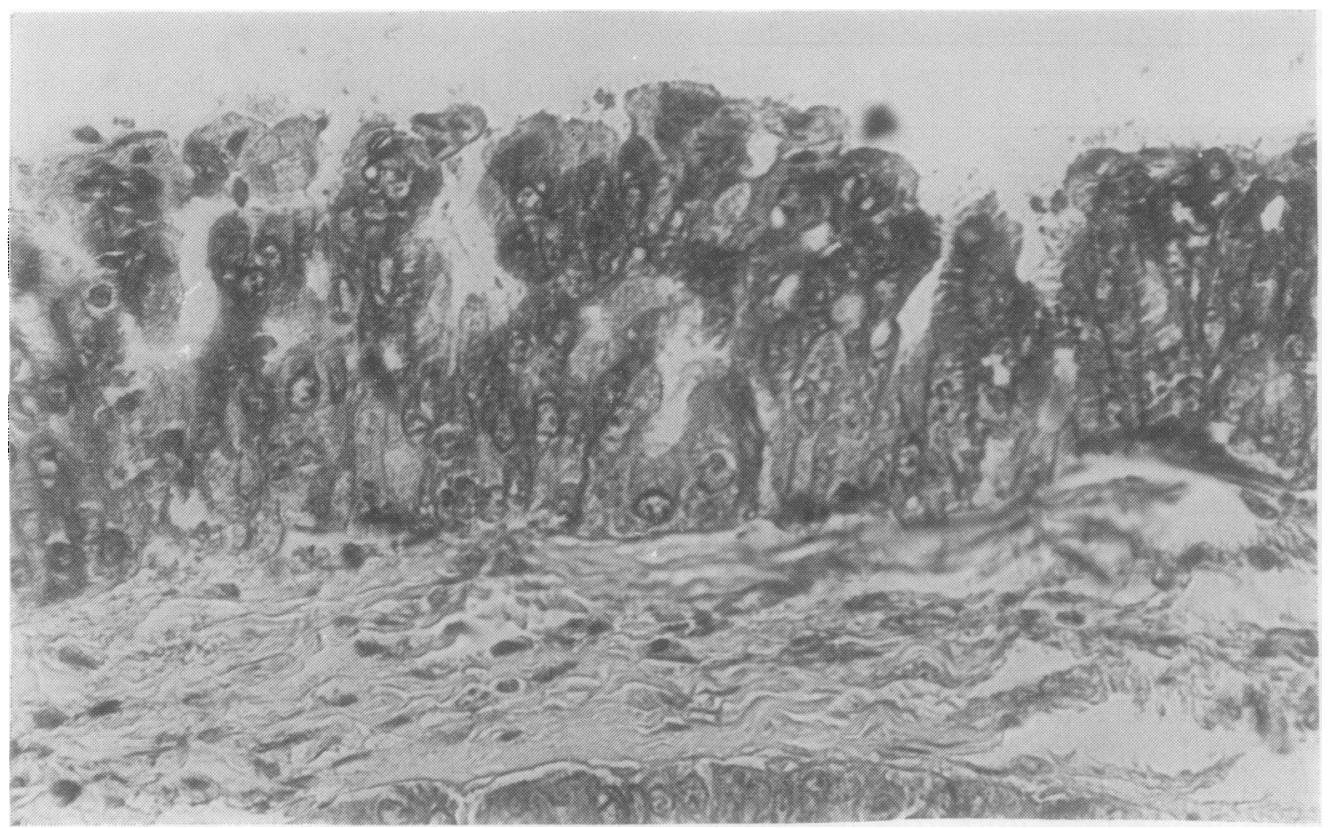

Fig. 1a $A$ section of a serous cystadenocarcinoma treated with anti-CEA antiserum at a dilution of 1 in 600; there is extensive staining of the cytoplasm of the neoplastic cells $(\times 305)$.

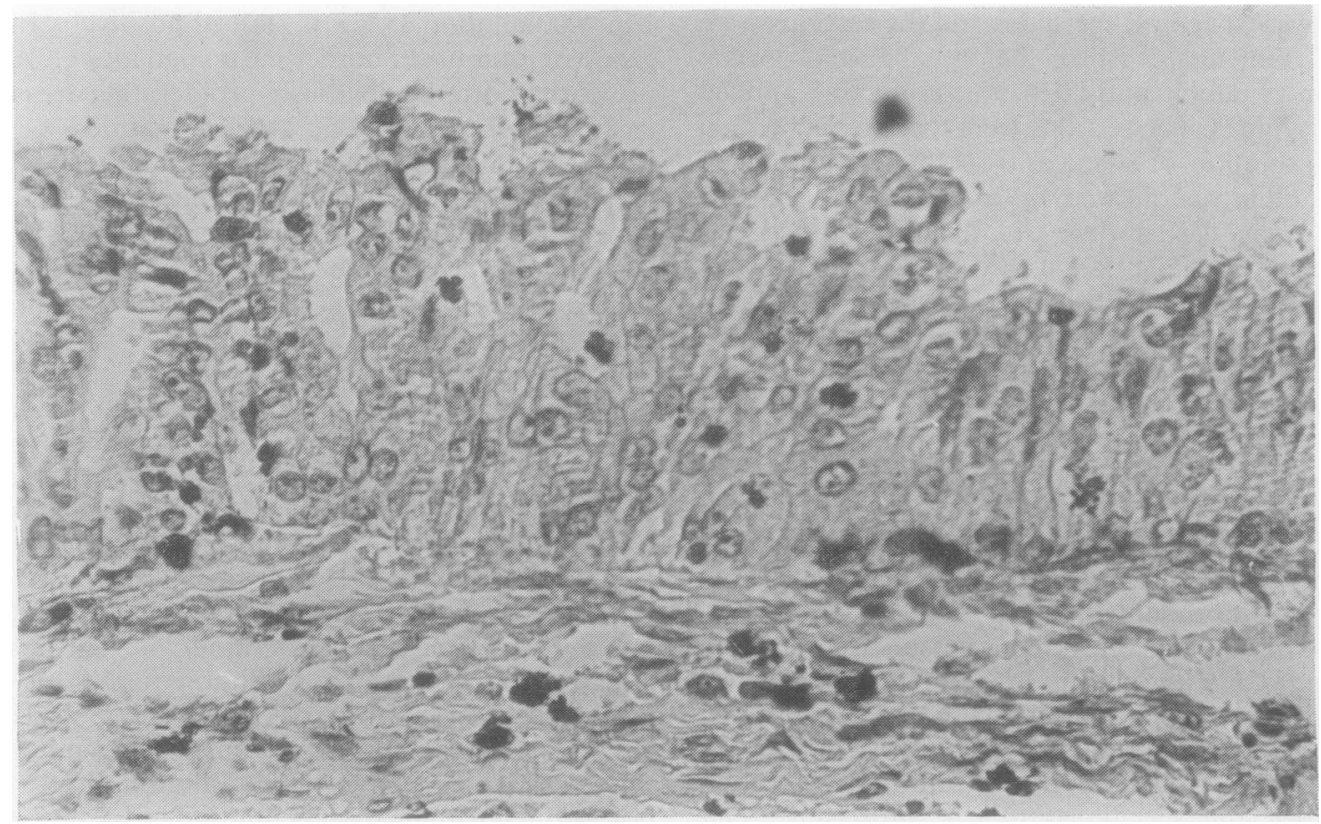

Fig. 1b A step section of the tissue shown in Fig. 1a after treatment with anti-CEA antiserum that had been absorbed with purified CEA: the cytoplasmic staining is completely abolished $(\times 305)$. 


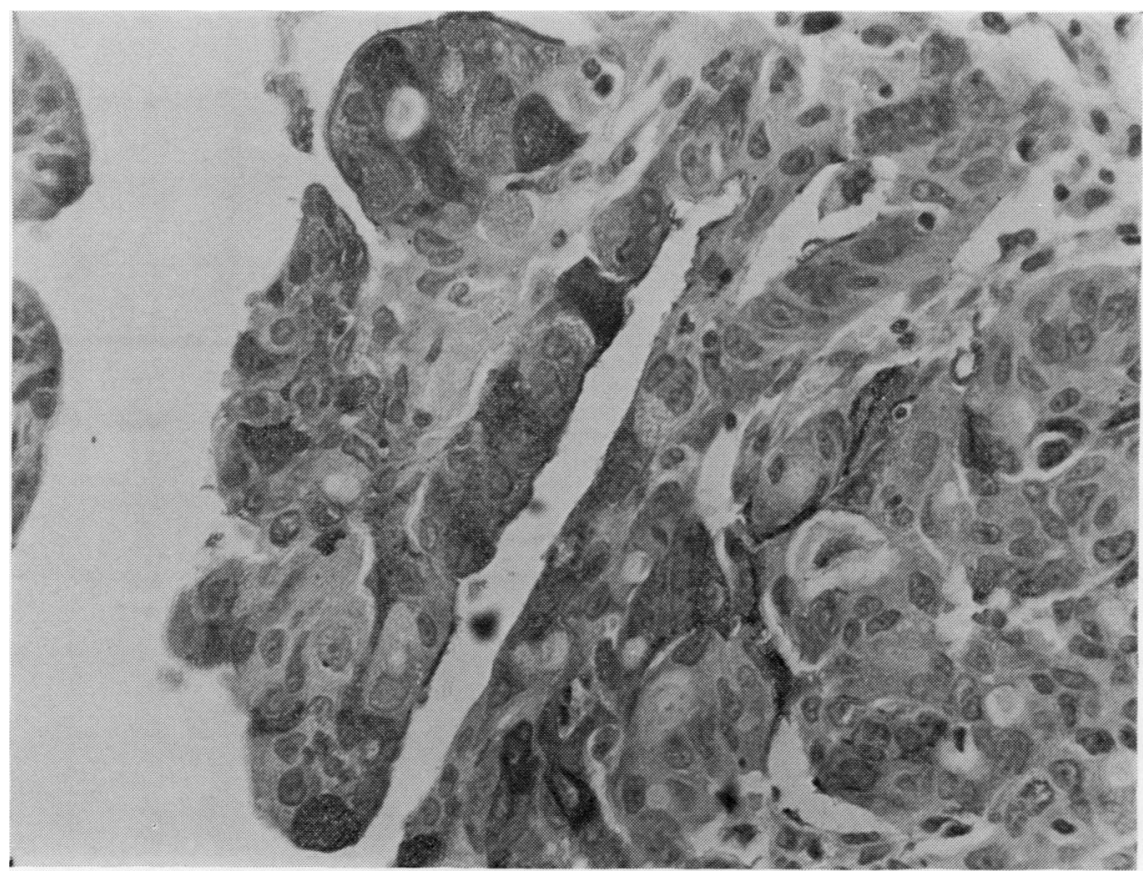

Fig. 2 An endometrioid adenocarcinoma stained for CEA; isolated cells show strong diffuse cytoplasmic staining $(\times 260)$.

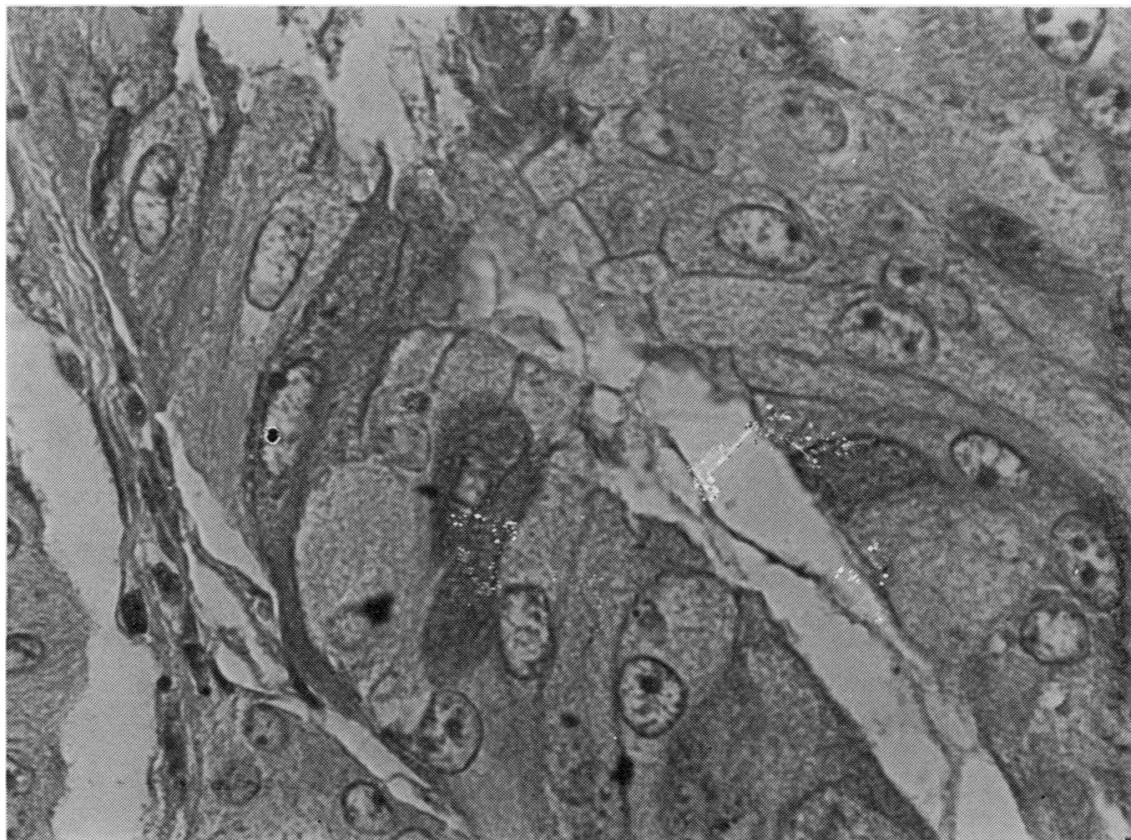

Fig. 3 An endometrioid adenocarcinoma stained for CEA; isolated cells show diffuse, but rather weak, cytoplasmic staining $(\times 490)$. 


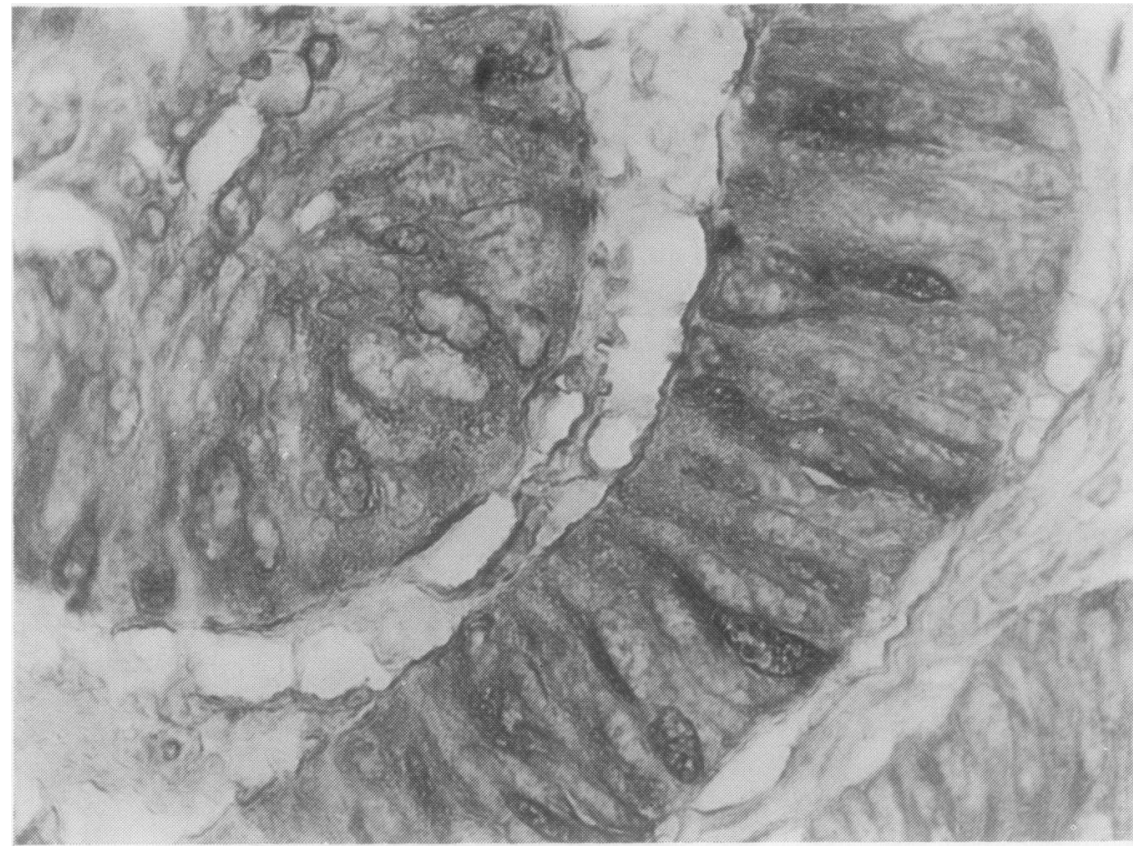

Fig. 4 A mucinous tumour of borderline malignancy stained for CEA; isolated cells show cytoplasmic staining maximal in the apical region $(\times 490)$.

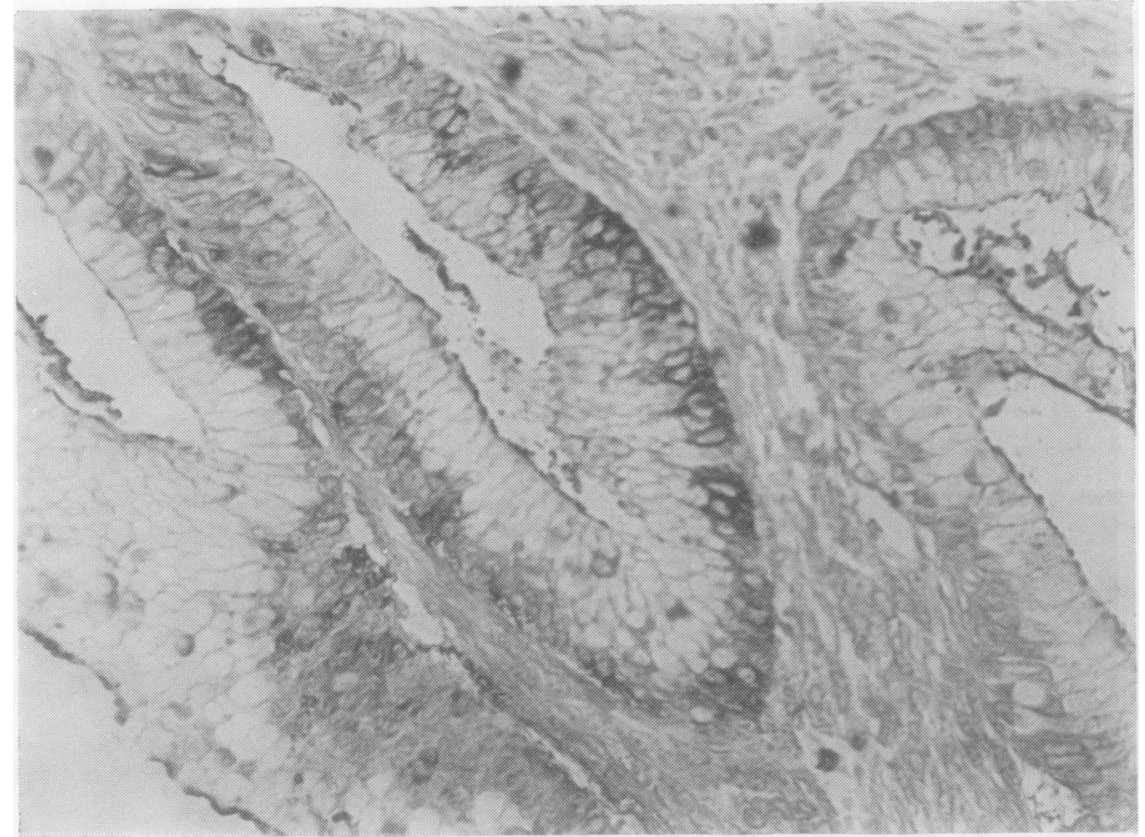

Fig. 5 A mucinous tumour of borderline malignancy stained for CEA; many cells show cytoplasmic staining maximal in the basal region $(\times 115)$. 


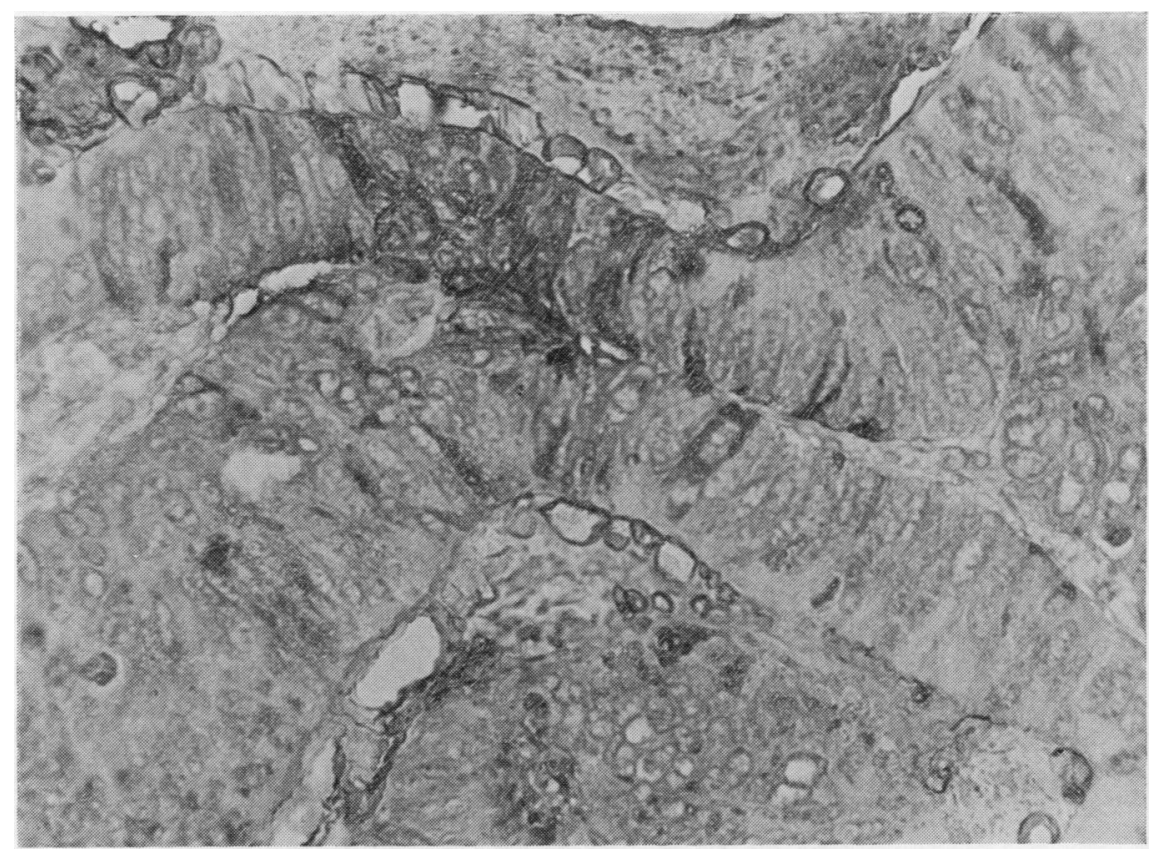

The remaining $20 \%$ of the malignant serous tumours continued to show marked staining up to a dilution of 1 in 1000, but at dilutions beyond this the staining was scanty and focal. As with the mucinous group of neoplasms those serous tumours considered to be of borderline malignancy stained positively for CEA both in the overtly proliferating epithelium and in that showing no atypical features. The serous carcinomas that lacked a content of CEA did not differ in any morphological manner, or in their degree of differentiation, from those which stained positively for this antigen.

\section{ENDOMETRIOID TUMOURS}

All 12 endometrioid tumours studied were malignant, and in these only the adenocarcinomatous areas were considered, staining in foci of metaplastic squamous epithelium being excluded. Three of the endometrioid tumours appeared to be devoid of CEA while the remaining nine showed no staining with antiserum dilutions of 1 in 1000 or greater.

\section{MESONEPHROID TUMOURS}

One mesonephroid tumour was completely negative, and another stained only focally, at a dilution of 1 in 100 . The other two neoplasms in this category stained at higher dilutions of the primary antiserum, one still giving a positive reaction for CEA at a dilution of 1 in 4000 .

\section{Discussion}

The first of the two questions posed at the outset of this study is readily answered. The synthesis of CEA is not a prerogative of any particular or specific variety of ovarian epithelial tumour and hence the presence of this antigen in a neoplasm of this type is of little value in differential histological diagnosis. It is true that serous tumours contain less CEA than do their mucinous counterparts but confusion between these two entities is uncommon and the frequently encountered diagnostic difficulty, that of differentiating serous from. endometrioid or mesonephric neoplasms, appears unlikely to be resolved by a study of tissue CEA content. It is also disappointing to note that the CEA content of a mucinous tumour was independent of the nature of the epithelium, appearing in similar quantities in both 'intestinal' and 'non-intestinal' type neoplasms.

The relationship between the degree of malignancy in epithelial ovarian tumours and their tissue CEA content is more difficult to evaluate. In mucinous tumours it is clear that, in general, malignant neoplasms contain considerably more CEA than do 


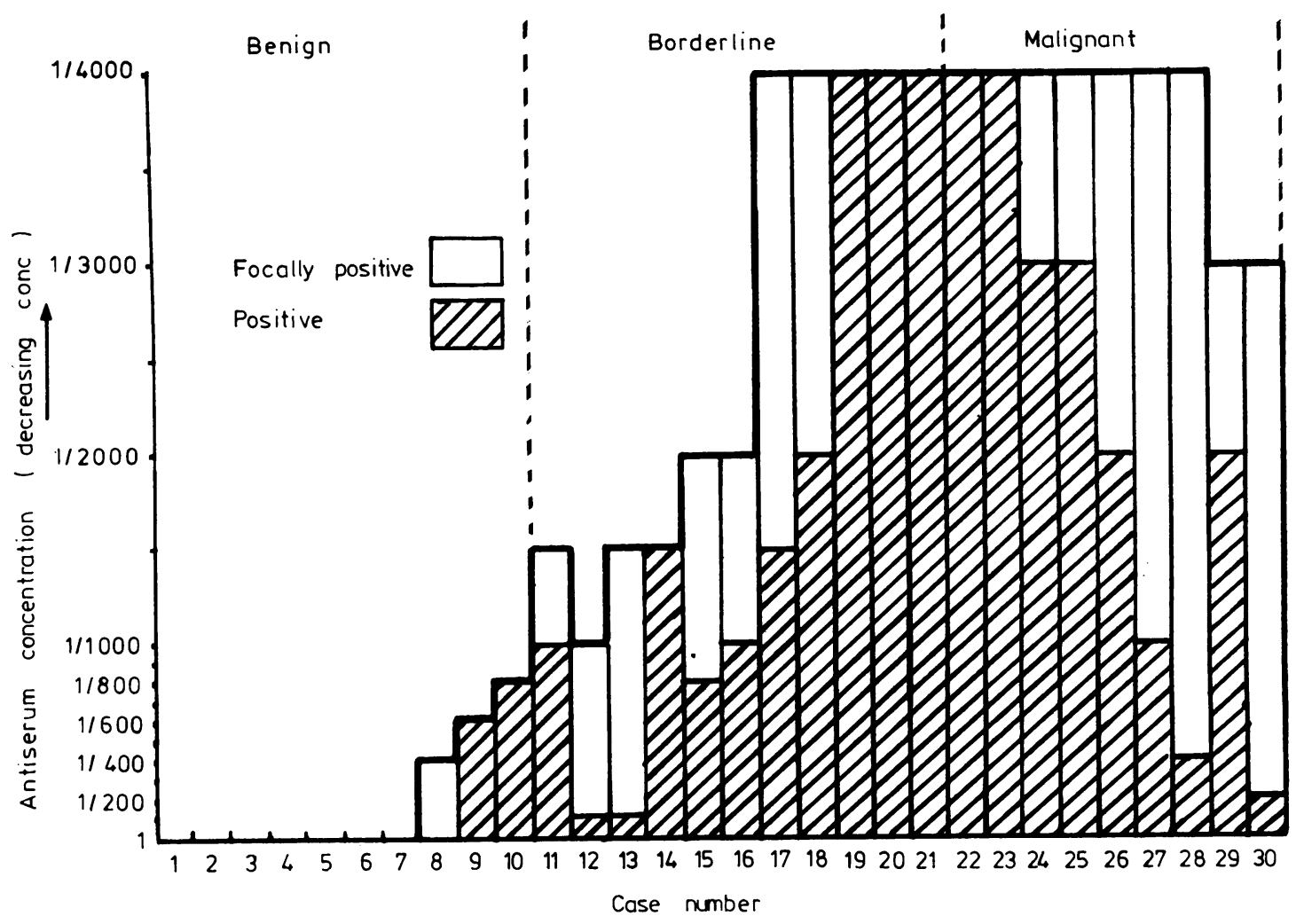

Fig. 7 Graphic representation of the reactions of mucinous tumours (M1-M30) at decreasing concentrations of anti-CEA antiserum. The CEA-positivity for each tumour is taken as the lowest antiserum concentration at which staining persists.

benign tumours but it would be an over-simplification to assert that there is a clearcut relationship between the degree of malignancy in mucinous tumours, as assessed histologically, and their tissue content of CEA. Thus some tumours of borderline malignancy contain as much CEA as do those which are overtly malignant while a proportion of histologically benign neoplasms have as high a CEA content as do those of borderline malignancy. The mucinous tumours can, as shown in Fig. 1, be arranged in rank and the findings interpreted in two ways. It could be argued that CEA content is a poor index of malignancy and does not allow for any clear separation either of benign from borderline or of borderline from malignant neoplasms; alternatively, it could be postulated that the demonstration of CEA content adds a degree of finesse to the histological definition of malignancy which necessitates a redivision of the groups. If this latter proposition is correct, then it could be suggested that the true division between the borderline and benign groups lies between cases M7 and M8 while that separating the borderline and malignant lies between cases M16 and M17. Our experience with case M11 suggests that a redivision on this basis may have some validity, for this tumour was originally considered to be benign and only because of its high content of CEA were further blocks taken, in one of which an area of atypical epithelial proliferation was found. It is therefore possible that a more exhaustive sampling of cases M8-10 would also have revealed similar borderline areas. The fact that significant quantities of CEA are found in both the non-proliferating and the proliferating areas of borderline tumours suggests, however, that synthesis of CEA and overt proliferative activity do not necessarily occur simultaneously, and it could therefore be postulated that the apparently benign tumours containing significant quantities of CEA were, in a biological or biochemical sense, already of borderline malignancy but had been 


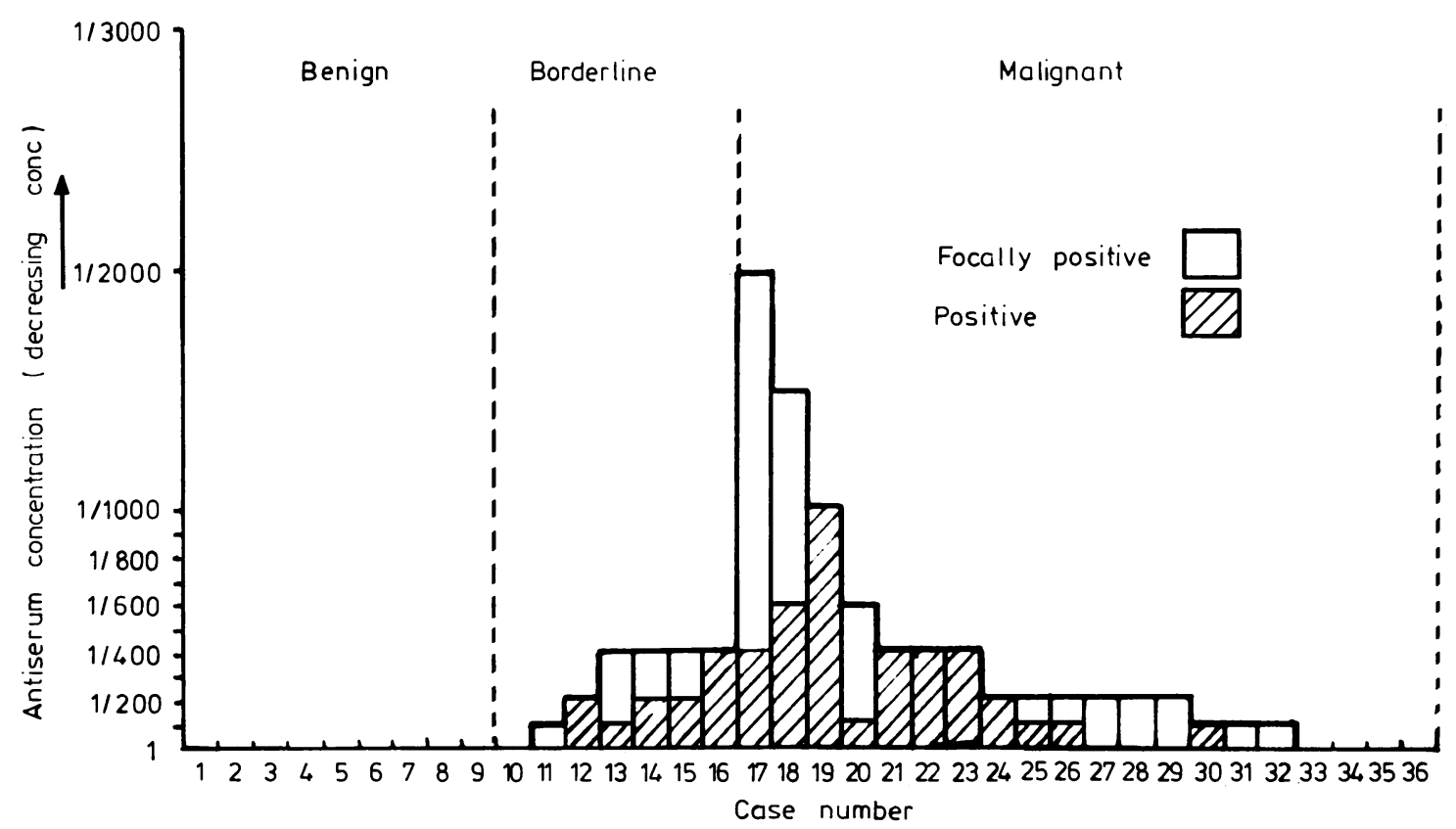

Fig. 8 Graphic representation of the reactions of serous tumours (S1-S36) at decreasing concentrations of anti-CEA antiserum. The CEA positivity for each tumour is taken at the lowest antiserum concentration at which staining persists.

removed before histological evidence of this potentiality for atypical growth had become apparent.

A significant proportion of the borderline group of mucinous tumours has a tissue CEA content which approximates to that found in frankly malignant tumours, and, applying the same arguments pursued above, it could be suggested that these neoplasms are already committed to become overtly malignant though as yet lacking all the histological criteria for the diagnosis of carcinoma. In this series, follow-up of the patients with borderline tumours was unsatisfactory and certainly inadequate to furnish any evidence either for or against this suggestion which can only be tested in a prospective study of a large series of patients with borderline tumours.

In the serous group of neoplasms the findings are less open to interpretation, speculative or otherwise. About the only conclusion that can be drawn from a study of tissue CEA content in this group of tumours is that a serous neoplasm containing CEA is probably malignant; the reverse contention, that tumours lacking CEA are therefore benign, is one that can not, however, be sustained.

\section{References}

Barrelet, V., and Mach, J. P. (1975). Variations of the carcinoembryonic antigen level in the plasma of patients with gynecologic cancers during therapy. American Journal of Obstetrics and Gynecology, 121, 164-168.

DiSaia, P. J., Haverback, B. J., Dyce, B. J., and Morrow, C. P. (1975). Carcinoembryonic antigen in patients with gynecologic malignancies. American Journal of Obstetrics and Gynecology, 121, 159-163.

Fox, H., Kazzaz, B., and Langley, F. A. (1964). Argyrophil and argentaffin cells in the female genital tract and in ovarian mucinous cysts. Journal of Pathology and Bacteriology, 88, 479-488.

Gold, P., and Freedman, S. O. (1965a). Demonstration of tumor-specific antigens in human colonic carcinomata by immunological tolerance and absorption techniques. Journal of Experimental Medicine, 121, 439-462.

Gold, P., and Freedman, S. O. (1965b). Specific carcinoembryonic antigens of the human digestive system. Journal of Experimental Medicine, 122, 467-481.

Goldenberg, D. M., Sharkey, R. M., and Primus, F. J. (1976). Carcinoembryonic antigen in histopathology: immunoperoxidase staining of conventional tissue sections. Journal of the National Cancer Institute, 57, 11-22.

Graham, R. C., Jr., and Karnovsky, M. J. (1966). The early stages of absorption of injected horseradish peroxidase in the proximal tubules of mouse kidney: ultrastructural cytochemistry by a new technique. Journal of Histochemistry and Cytochemistry, 14, 
291-298.

Hansen, H. J., Snyder, J. J., Miller, E., Vandevoorde, J. P., Miller, O. N., and Burns, J. J. (1974). Carcinoembryonic antigen (CEA) assay, a laboratory adjunct in the diagnosis and management of cancer. Human Pathology, 5, 139-147.

Heyderman, E., and Neville, A. M. (1977). A shorter immunoperoxidase technique for the demonstration of carcinoembryonic antigen and other cell products. Journal of Clinical Pathology, 30, 138-140.

Isaacson, P., and Le Vann, H. P. (1976). The demonstration of carcinoembryonic antigen in colorectal carcinoma and colonic polyps using an immunoperoxidase technique. Cancer, 38, 1348-1356.

Khoo, S. K., and Mackay, E. V. (1973). Carcinoembryonic antigen in cancer of the female reproductive system: sequential levels and effects of treatment. Australian and New Zealand Journal of Obstetrics and Gynaecology, 13, 1-7.

Lo Gerfo, P., Krupey, J., and Hansen, H. J. (1971). Demonstration of an antigen common to several varieties of neoplasia. New England Journal of Medicine, 285, 138-141.

Marchand, A., Fenoglio, C. M., Pascal, R., Richart, R. M., and Bennett, S. (1975). Carcinoembryonic antigen in human ovarian neoplasms. Cancer Research, 35, 3807-3810.

Primus, F. J., Wang, R. H., Sharkey, R. M., and Goldenberg, D. M. (1975). Detection of carcinoembryonic antigen in tissue sections by immunoperoxidase. Journal of Immunological Methods, 8, 267-275.

Puri, S., Mesa-Tejada, R., Husami, N., Bennett, S., Richart, R., and Fenogolio, C. M. (1977). Carcinoembryonic antigen in gynecologic patients. 1. Correlation of plasma levels and tissue localization. Gynecologic Oncology, 5, 331-337.

Pusztaszeri, G., and Mach, J. P. (1973). Carcinoembryonic antigen (CEA) in non-digestive cancerous and normal tissues. Immunochemistry, 10, 197-204.

Reynoso, G., Chu, T. M., Holyoke, D., Cohen, E., Nemoto, T., Wang, J. J., Chuang, J., Guinan, P., and Murphy, G. P. (1972). Carcinoembryonic antigen in patients with different cancers. Journal of the American Medical Association, 220, 361-365.

Rutanen, E. M., Lindgren, J., Sipponen, P., Stenman, U. H., Saksela, E., and Seppälä, M. (1978). Carcinoembryonic antigen in malignant and nonmalignant gynecologic tumors: circulating levels and tissue localization. Cancer, 42, 581-590.

Samaan, N. A., Smith, J. P., Rutledge, F. N., and Schultz, P. N. (1976). The significance of measurement of human placental lactogen, human chorionic gonadotropin, and carcinoembryonic antigen in patients with ovarian carcinoma. American Journal of Obstetrics and Gynecology, 126, 186-189.

Serov, S. F., and Scully, R. E. (1973). Histological Typing of Ovarian Tumours (International Histological Classification of Tumours, No. 9). World Health Organisation, Geneva.

Sternberger, L. A., Hardy, P. H., Jr., Cuculis, J. J., and Meyer, H. G. (1970). The unlabelled antibody enzyme method of immunohistochemistry. Preparation and properties of soluble antigen-antibody complex (horseradish peroxidase-antihorseradish peroxidase) and its use in identification of spirochetes. Journal of Histochemistry and Cytochemistry, 18, 315-333.

van Nagell, J. R., Jr., Donaldson, E. S., Gay, E. C., Sharkey, R. M., Rayburn, P., and Goldenberg, D. M. (1978). Carcinoembryonic antigen in ovarian epithelial cystadenocarcinomas: the prognostic value of tumor and serial plasma determinations. Cancer, 41, 23352340.

van Nagell, J. R., Jr., Meeker, W. R., Parker, J. C., Jr., and Harralson, J. D. (1975a). Carcinoembryonic antigen in patients with gynecologic malignancy. Cancer, 35, 1372-1376.

van Nagell, J. R., Jr., Pletsch, Q. A., and Goldenberg, D. M. (1975b). A study of cyst fluid and plasma carcinoembryonic antigen in patients with cystic ovarian neoplasms. Cancer Research 35, 1435-1437.

Requests for reprints to: Professor H. Fox, Department of Pathology, University of Manchester, Stopford Building, Oxford Road, Manchester M13 9PT, UK. 